The Egyptian Journal of Hospital Medicine (January 2022) Vol. 86, Page 486-492

\title{
Enucleation Versus Standard Pancreatic Resection for Benign Lesions and Borderline Tumors: A Comparative Study
}

\author{
Ashraf Mohammad El-Badry, Ahmed Gaber Mahmoud, Mohamed Mahmoud Ali*
}

Department of Surgery, Faculty of Medicine, Sohag University, Sohag, Egypt

*Corresponding author: Mohamed Mahmoud Ali, Mobile: (+20)1004564240, E-mail: drmohamedmahmoud1969@gmail.com

\begin{abstract}
Background: Enucleation of pancreatic benign lesions and borderline tumors, compared with standard pancreatic resection, may avoid postoperative impairment of the metabolic pancreatic functions. However, its influence on postoperative morbidity and disease recurrence seems obscure. Therefore, the choice between both approaches remains controversial.Objective: To evaluate the outcome of enucleation compared with standard pancreatic resection (SPR) of pancreatic benign lesions and borderline tumors among patients presented to Sohag University Hospital. Patients and Methods: Adult patients who underwent enucleation (group A) versus standard resections (group B) of pancreatic benign lesions and borderline tumors at Sohag University Hospital (June 2017 - May 2021) were prospectively enrolled. Both surgical techniques were compared regarding their influence on postoperative metabolic functions of the pancreas, surgical complications, and disease recurrence. Results: Sixteen patients (eight per group) with comparable gender and age distribution were eligible. Group A had significantly shorter operative time, lower amounts of intra-operative blood loss and less transfusions compared to group B. Likewise, patients in group A exhibited significantly reduced severity of surgical complications, including postoperative pancreatic fistula, and required significantly shorter periods of hospital stay. The incidence of new onset diabetes mellitus and pancreatic exocrine insuffficiency were significantly lower among patients in group A compared with those in group B. During follow-up, no recurrence was found in both groups. Conclusion: It could be concluded that enucleation of pancreatic benign lesions and borderline tumors preserves pancreatic metabolic functions, reduces postoperative morbidity and confers satisfactory oncologic outcome.
\end{abstract}

Keywords: Enucleation, Pancreatectomy, Pancreatic cysts, Pancreatic fistula

\section{Introduction:}

Presurgical distinction between pancreatic benign lesions (non-neoplastic, pseudotumors) and borderline tumors from pancreatic adenocarcinoma remains challenging (1). The diagnostic difficulty is related mainly to their imaging features which frequently overlap with those of adenocarcinoma in addition to lack of pathognomonic biochemical markers ${ }^{(2)}$.

In Egypt, pancreatic cancer ranks eleventh among all types of malignant diseases, probably due delayed diagnosis. Therefore, the Global Cancer Observatory report does not describe pancreas cancer among common malignant diseases affecting Egyptians (3). Subsequently, pancreatic benign lesions and even borderline tumors are not frequently discovered, given the scarcity and late presentation of the majority of cases ${ }^{(4)}$. A variety of benign pancreatic lesions may grossly appear intra-operatively as well-demarcated zone of parenchymal fibrosis and retention cysts as a result of chronic pancreatitis. Other benign lesions include inflammatory masses and pseudotumors, lymphoepithelial and epidermoid cysts and pancreatic hamartomas, hemangiomas and lymphangiomas ${ }^{(5-8)}$. These benign diseases may be designated as pancreatic tumor-like lesions or pseudotumors ${ }^{(6,8,9)}$.

According to the World Health Organization classification, benign cyctic pancreatic tumors entail serous cystadenoma, mucinous cystadenoma, intraductal papillary mucinous adenoma and mature teratoma. Borderline pancreatic tumors include mucinous cystic neoplasm, intraductal papillary mucinous neoplasm and solid pseudopapillary neoplasm ${ }^{(\mathbf{1 0 - 1 3 )}}$. Furthermore, villous adenoma of the pancreatic duct is a rare premalignant pancreatic lesion (14). Prophylactic excision of villous adenoma is beneficial because about $50 \%$ of cases would harbour foci of adenocarcinoma ${ }^{(\mathbf{1 5})}$.

Due to the common preoperative diagnostic uncertainty, selection of enucleation versus standard pancreatic resection (SPR) to remove doubtful pancreatic lesions remains unsettled ${ }^{(16-18)}$.

To address this complex subject, we prospectively evaluated the outcome of enucleation compared with SPR of pancreatic benign lesions and borderline tumors among patients presented to Sohag University Hospital. Both techniques were compared regarding postoperative quality of pancreatic metabolic functions, surgical complications and disease recurrence.

\section{PATIENTS AND METHODS}

This prospective study included a total of sixteen patients with pancreatic benign lesions and borderline tumors, attending at Sohag University Hospitals. This study was conducted between June 2017 - May 2021.

Exclusion criteria: age $<18$ years, histologically proven pancreatic adenocarcinoma, presence of distant metastasis and recurrent lesions.

According to the surgical treatment strategy, patients were divided into two equal groups, 8 each, 
group A (enucleation) versus group B (standard pancreatic resection, SPR). Allocation of patients to either group was based on preoperative evaluation by multidisciplinary team.

All patients underwent preoperative work-up including meticulous clinical assessment, medical imaging, and estimation of relevant laboratory markers. Imaging studies comprised abdominal ultrasonography and contrast-enhanced computed tomography of the abdomen and chest. Abdominal magnetic resonance imaging and esophagogastroduodenoscopy were carried out selectively as advised by the multidisciplinary team. In addition to routine laboratory investigations, blood levels of carbohydrate antigen (CA 19:9) and carcinoembryonic antigen (CEA) were measured in all patients. Endoscopic ultrasound and sampling of the pancreatic lesions were performed in a few patients who were able to afford this examination since it was not regularly available in our hospital. Pancreaticoduodenectomy (Whipple procedure) was intentionally performed in patients who had preoperative diagnosis (by endoscopic retrograde cholangio-pancreatography) of villous adenoma (and hence they were included in group B) due to the reportedly high risk of undetected adenocarcinoma in the tiny endoscopic tissue samples (15)

\section{Definition of outcomes:}

Clinically relevant postoperative pancreatic fitula (POPF) was defined according to the International Study Group of Pancreatic Surgery (ISGPS) as drain output with amylase level $>3$ times the upper normal value of serum amylase and clinical events directly related to POPF ${ }^{(\mathbf{1 9})}$. Thus, POPF grade A represents just biochemical leak, grade $B$ denotes modification of postoperative care (maintenance abdominal drains in place for $>3$ weeks or repositioning by endoscopic or percutaneous interventions) while grade $\mathrm{C}$ refers to re-laparotomy, organ failure (single or multiple) or death in direct relation to $\mathrm{POPF}^{(16)}$.

Delayed Gastric Emptying (DGE) was also diagnosed along with ISGPS definitions ${ }^{(20)}$. Common presentations of DGE entail high drainage from nasogastric tube, vomiting and intolerance to enteral feeding. DGE is graded in proportion to the period of postoperative nasogastric intubation as follows: grade A (mild DGE) 4-7 days, grade B (moderate DGE) 8-14 days, grade $\mathrm{C}$ (severe DGE) $>14$ days ${ }^{(20)}$.

Criteria of pancreatic exocrine insufficiencey, according to Beger and his co-workers ${ }^{(17)}$, comprise postoperative steatorrhea, need of enzyme supplementation, and increase of body weight following supplemental enzyme administration. Endocrine insufficiency was defined as deveolpment of postoperative new onset diabetes mellitus (NODM) in preoperatively normoglycemic patients ${ }^{(\mathbf{1 7})}$.

\section{Ethical Consideration:}

An approval of the study was obtained from Sohag University academic and ethical committee. Every patient signed an informed written consent for acceptance of the operation. This work has been carried out in accordance with The Code of Ethics of the World Medical Association (Declaration of Helsinki) for studies involving humans.

\section{Statistical analysis}

We used Graphpad Prism 7 for statistical comparison betweenn groups. Qualitative data were shown as numbers and percentages whereas quantitative records were expressed as median and range. Student t-test was utilized to compare quantitative variables. Differences between groups were considered statistically significant with $\mathrm{p}$ value < 0.05 .

\section{RESULTS}

Sixteen eligible patients were enrolled in the study (eight per group). Demographic data, clinical characteristics and laboratory findings were almost similar in both groups (table 1). Indications of surgery were either pancreatic benign lesions or borderline tumors in various anatomical portions of the pancreas. All patients in group A underwent enucleation whereas different types of SPRs were carried out in group B including pancreatico-duodenectomy, central and distal pancreatectomy.

The most common lesions were pancreatic neuroendocrine tumor and serous cystadenoma (3 cases each) followed by mucinous cystadenoma (2), infected pancreatic pseudocyst (2) and villous adenoma of the pancreatic duct (2). The remaining 4 cases comprised solid pseudopapillary tumor, inflammatory mass, lymphoepithelial cyst and traumatic organized hematoma. Indications of surgery, location of pancreatic lesions and type of surgical procedure are listed in table 2.

Enucleation (figures 1 and 2) of benign and borderline pancreatic lesions (group A) required significantly shorter operative time than SPR (group B). Likewise, the amounts of blood loss and intraoperative transfusions were significantly lower in group A compared with group $\mathrm{B}$, table 3 . Postoperatively, histopathologic assessment confirmed negative gross and microscopic margins of the excised specimens in all patients of both groups. Overall, postoperative complications were significantly less severe in group A compared with group B. In group A, only mild transient pancreatic leak (grade "A" POPF) occurred in one patient who underwent enucleation of serous cystadenoma located in the pancreatic body. Spontaneous resolution occurred within 12 days under conservative treatment. One patient suffered from paralytic ileus which responded adequately to nasogastric decompression and medical treatment. Another one patient had wound infection requiring local debridement. Contrarily, clinically relevant 
grades (B and C) of POPF occured in 2 among 8 patients $(25 \%)$ included in group B after Whipple procedure and distal pancreatectomy, respectively. In the former patient, treatment with ultrasonographyguided percutaneous drainage was sufficient. The other patient developed signs of sepsis and underwent relaparotomy for drainage of intraperitoneal collection, revision of pancreatic stump and peritoneal wash-out. Wound infection occurred in 2 patients in whom bedside wound opening, drainage and local antiseptics were necessary. Mild grade of delayed gastric emptying (grade A) was observed in one patient after Whipple procedure and was conservatively managed by continuous nasogastric drainage for 6 days. One patient suffered from postoperative chest infection which was successfully treated with broad spectrum systemic antibiotics. In contrast to group B, patients comprising group A required significantly shorter time until removal of abdominal drains as well as

Table (1): Demographics and preoperative data. significantly reduced length of hospital saty, table 4 . Of note, there was no mortality among the entire study population.

Impairement of the metabolic functions of the pancreas was limited to SPR group. Manifestations of exocrine insufficiency that required enzymatic supplementation occurred exclusively in group B (3 patients, 37.5\%) following Whipple procedure in 2 patients and distal pancreatectomy in one. NODM occurred also entirely in group B in 2 among 7 patients (28.6\%) who had no history of preoperative diabetes mellitus. In sharp contrast, there was no evidence of metabolic pancreatic insufficiency in relation to enucleation (group A).

To evaluate the rate of recurrent disease after enucleation versus SPR, follow up was regularly maintained (median: 31, range: 3- 51 months). During this period there was no clinical or radiological evidence of recurrent disease in both groups.

\begin{tabular}{|c|c|c|}
\hline & Group A & Group B \\
\hline \multicolumn{3}{|c|}{ Preoperative characteristics* } \\
\hline - Female gender (n) & $5 / 8(62.5 \%)$ & $6 / 8(75 \%)$ \\
\hline - $\operatorname{Age}^{\S}$ (year) & $42(25-66)$ & $(4332-70)$ \\
\hline$-\mathrm{BMI}^{\S}\left(\mathrm{kg} / \mathrm{m}^{2}\right)$ & $28(22-32)$ & $27(23-34)$ \\
\hline \multicolumn{3}{|l|}{ - History of: } \\
\hline - $\quad$ Smoking & $2 / 8(25 \%)$ & $2 / 8(25 \%)$ \\
\hline - Diabetes mellitus & $1 / 8(12.5 \%)$ & $1 / 8(12.5 \%)$ \\
\hline \multicolumn{3}{|l|}{ Tumor markers ${ }^{\S *}$} \\
\hline - CA 19:9 (U/mL) & $7(4.5-12)$ & $9(3-23)$ \\
\hline - CEA (ng/mL) & $0.9(0.3-4)$ & $1.5(0.5-5)$ \\
\hline
\end{tabular}

Table (2): Indications of surgery, location of pancreatic lesions and surgical procedures

\begin{tabular}{|c|c|c|c|c|}
\hline n. & อ̊ํำ & $\begin{array}{c}\text { Presumptive } \\
\text { diagnosis } \\
\text { (preoperatively) }\end{array}$ & Location & $\begin{array}{c}\text { Surgical } \\
\text { procedure }\end{array}$ \\
\hline 1 & \multirow{8}{*}{ 岂 } & Solid pseudopapillary tumor & Head & Enucleation \\
\hline 2 & & Neuroendocrine tumor & Head & Enucleation \\
\hline 3 & & Indeterminate $^{*}$ & Neck & Enucleation \\
\hline 4 & & Neuroendocrine tumor & Body & Enucleation \\
\hline 5 & & Serous cystadenoma & Body & Enucleation \\
\hline 6 & & Neuroendocrine tumor & Body & Enucleation \\
\hline 7 & & Indeterminate $^{* *}$ & Body & Enucleation \\
\hline 8 & & Mucinous cystadenoma & Body & Enucleation \\
\hline 9 & \multirow{8}{*}{ ڤُ } & Villous adenoma ${ }^{* * *}$ & Head & $\mathrm{PD}^{\S \S}$ \\
\hline 10 & & Villous adenoma ${ }^{* * *}$ & Head & $\mathrm{PD}^{\S \S}$ \\
\hline 11 & & Hematoma (traumatic) ${ }^{\S}$ & Neck & $\mathrm{CP}^{\S \S}$ \\
\hline 12 & & Neuroendocrine tumor & Body & DP \\
\hline 13 & & Infected pseudocyst & Body & DP \\
\hline 14 & & Infected pseudocyst & Body & DP \\
\hline 15 & & Mucinous cystadenoma & Body \& tail & $\mathrm{DP}+\mathrm{S}$ \\
\hline 16 & & Serous cystadenoma & Body \& tail & $\mathrm{DP}+\mathrm{S}$ \\
\hline
\end{tabular}

$\mathrm{n}$, patient number; PD, pancreaticoduodenectomy (Whipple procedure); CP, central pancreatectomy; DP, distal pancreatectomy; S, splenectomy; *history of chronic pancreatitis, definitive diagnosis: inflammatory mass; ${ }^{* *}$ definitive diagnosis: lymphoepithelial cyst; ***preoperatively assumed by endoscopic biopsy (endoscopic retrograde cholangiopancreatography), definitive diagnosis: villous adenoma with adenocarcinomatous foci; ${ }^{\S}$ associated with with rupture of the pancreatic duct; ${ }^{\S \S}$ pancreatico-digestive anastomosis technique: pancreatico-gastrostomy. 
Table (3): Operative data, postoperative abdominal drainage and length of hospital stay

\begin{tabular}{lcc}
\hline & \multicolumn{1}{c}{ Group A } & Group B \\
\hline Operative time $(\text { minutes })^{*}$ & $140(90-230)$ & $250(110-375)$ \\
Blood loss $(\mathrm{ml}) *^{\S}$ & $300(100-800)$ & $500(300-1200)$ \\
Transfusions: & & \\
$-\quad$ Packed RBCs $(\mathrm{U})^{* \S}$ & $1(0-2)$ & $3(0-4)$ \\
$-\quad$ FFP $(\mathrm{U}) * \S$ & $1(0-2)$ & $4(2-8)$ \\
\hline
\end{tabular}

" significant difference $(\mathrm{p}<0.05) ;{ }^{\S}$ median (range); $\mathrm{m}$, minute; ml, milliliter;

FFP, fresh frozen plasma; RBCs, red blood cells

Table (4): Postoperative complications and their management

\begin{tabular}{|c|c|c|c|c|c|}
\hline n. & 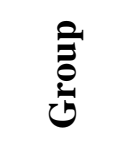 & Highest complication & Management & $\begin{array}{c}\text { Abdominal } \\
\text { drainage } \\
\text { (days)* }^{*}\end{array}$ & $\begin{array}{l}\text { Hospital } \\
\text { stay } \\
(\text { days) }\end{array}$ \\
\hline 1 & \multirow{8}{*}{ 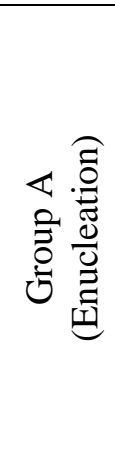 } & None & None & \multirow{8}{*}{$\overbrace{\substack{0 \\
j}}^{+\infty}$} & \multirow{8}{*}{$\overbrace{\substack{c \\
c}}^{\substack{c \\
\infty}}$} \\
\hline 2 & & Minor POPF & Conservative & & \\
\hline 3 & & Wound infection & Debridement & & \\
\hline 4 & & None & None & & \\
\hline 5 & & Paralytic ileus & Conservative & & \\
\hline 6 & & None & None & & \\
\hline 7 & & None & None & & \\
\hline 8 & & None & None & & \\
\hline 9 & \multirow{8}{*}{ 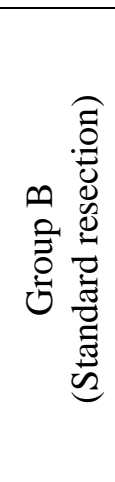 } & POPF & Drainage $^{* * *}$ & \multirow{8}{*}{ 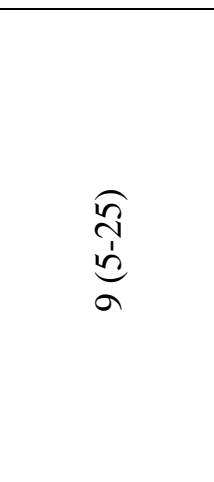 } & \multirow{8}{*}{ 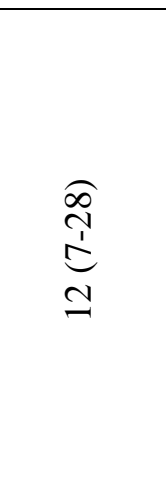 } \\
\hline 10 & & DGE & Conservative & & \\
\hline 11 & & Wound infection & Debridement & & \\
\hline 12 & & None & None & & \\
\hline 13 & & Wound infection & Debridement & & \\
\hline 14 & & POPF & Relaparotomy ${ }^{* * *}$ & & \\
\hline 15 & & Pneumonia & Conservative & & \\
\hline 16 & & None & None & & \\
\hline $\begin{array}{l}\mathrm{n}, \mathrm{p} \\
\text { panc } \\
\text { days }\end{array}$ & $\begin{array}{l}\text { nul } \\
\mathrm{c} \text { abs } \\
\text { iired } \\
\text { tal sta }\end{array}$ & $\begin{array}{l}\text { er; median (range); } \\
\text {, peritoneal lavage and } \\
\text { abdominal drainage co } \\
\text { ompared with group B }\end{array}$ & $\begin{array}{l}\text { rcutaneous drain } \\
\text { vision of the panc } \\
\text { ared with group I } \\
0.05 \text { ) }\end{array}$ & $\begin{array}{l}\text { ound guidec } \\
\text {; }{ }^{\S} \text { significan } \\
{ }_{\S} \text { significant }\end{array}$ & $\begin{array}{l}\text { drainage of } \\
\text { s number of } \\
\text { ter duration }\end{array}$ \\
\hline
\end{tabular}



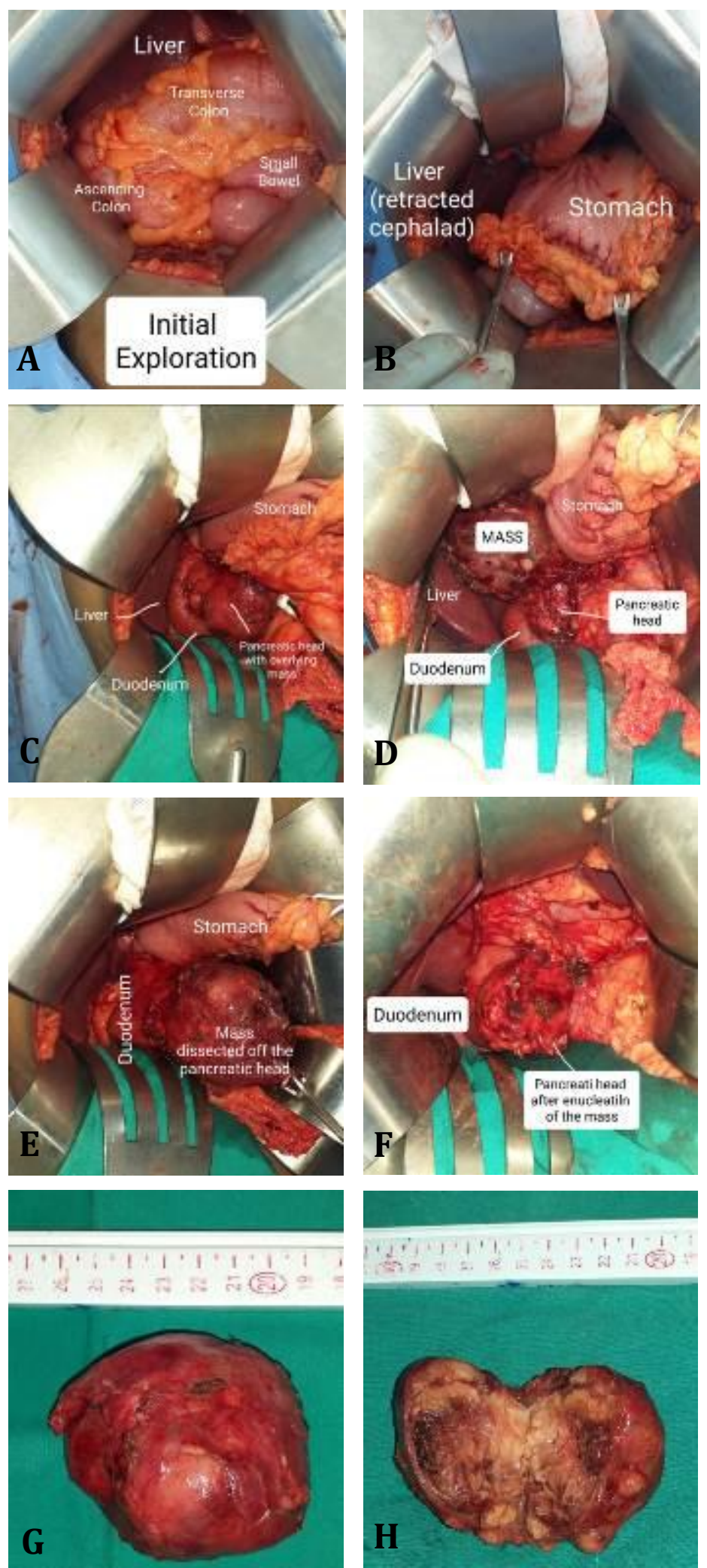

Figure (1): Enucleation of solid pseudopapillary tumor from pancreatic head: A) Initial exploration. B) Cephalad retraction of the liver. C) Opening of the lesser sac and exposure of pancreatic head tumor. D) Dissection of the tumor from the pancreatic head. E) Almost completed enucleation of the tumor. F) The pancrreatic head after complete enucleation of the tumor. G) The enucleated tumor. H) The enucleated tumor, bisected. 

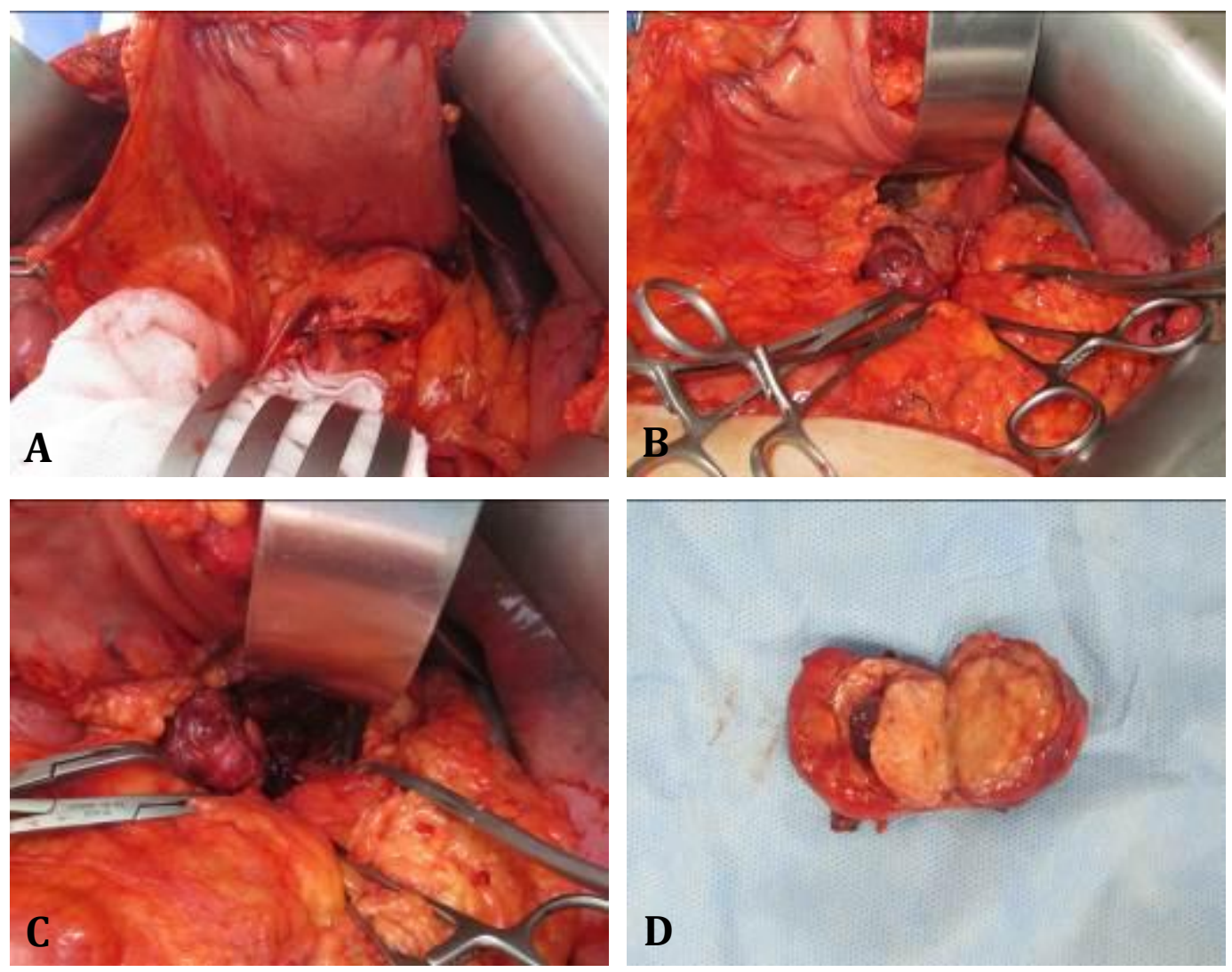

Figure (2): Enucleation of neuroendocrine tumor from the pancreatic body: A) Anterior protrusion of the pancreatic body surface due to posterioly-located tumor. B) Dissection of the pancreatic body tumor. C) Enucleation of pancreatic body mass, almost completed. D) The enucleated tumor, bisected.

\section{DISCUSSION}

In this study we addressed the controversial views of enucleation versus SPR of pancreatic lesions that have been preoperatively assumed as benign lesions or border-line tumors. Compared with SPR, we found that enucleation appears to efficiently preserve the metabolic functions of the pancreas without compromising the oncologic outcome. Moreover, enucleation conferred protection against severe postoperative complications.

In essence, SPR would certainly eradicate any suspicious pancreatic lesion (9, 21-23). However, postoperative histopathologic assessment could ultimately confirm a benign lesion or borderline tumor. In this scenario, SPR could have led to unnessecary loss of substantial pancreatic parenchymal mass with needless impairment of pancreatic endocrine and exocrine functions $\left(\mathbf{1 7},{ }^{24}\right)$. On the other hand, enucleation represents a tissue-sparing technique that may combine the advantages of excision of doubtful pancreatic lesion and preservation of the organ's

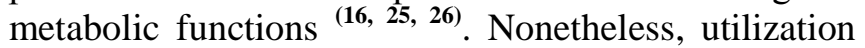
of this strategy could be associated with increased postoperative complications, particularly POPF, compared with standard pancreatic resections (27). Therefore, we compared both surgical strategies, enucleation versus SPR, regarding postoperative metabolic functions, surgical complications, and recurrence.

We found that enucleations were carried out in remarkably shorter operative time with decreased blood loss compoared with SPR. Similar data were previously reported in the setting of enucleation of pancreatic neuroendocrine tumors and solid pseudopapillary neoplasms (25, 26). In our series, impairment of exocrine pancreatic functions affected more than one third of patients after SPR. In sharp contrast, patients who received enucleation did not develop exocrine insufficiency. This was in agreement with previous reports indicating that, contrary to SPR, enucleation is associated with protection against postoperative pancreatic exocrine insufficiency ${ }^{(25,28)}$.

Likewise, the incidence of NODM postoperatively was $28.6 \%$, and was limited to group B after distal pancreatectomy. In the same line, previous literature provides strong evidence on decreased incidence of postoperative NODM following pancreatic enucleation compared with SPR $(17,27)$

Postoperative complications were thoroughly assessed in both groups. We found that enucleation was associated with reduced severity of postoperative complications, including POPF. This is particularly relevant because of the remarkably contradictory results reported by previous studies ${ }^{(25-27)}$. 
After excision of the pancreatic lesion, irrespective of the applied surgical technique, we have constantly removed at least half centimeter of additional pancreatic tissues as safety margin from the pancreatic bed. Thus, obtaining histologically proven negative margins in all patients was associated with zero recurrence rate in both groups. Our results support previous data indicating that enucleation with negative margins confers acceptable oncological outcome ${ }^{\mathbf{( 2 8 , 2 9 )}}$.

It should be underlined that the small number of patients represents a shortcoming in our study. However, given the generally low rates of early diagnosis of pancreatic lesions, our study may shed a spotlight on the lines of management of a relatively rare group of pancreatic lesions.

\section{CONCLUSION}

Enucleation of benign lesions and borderline tumors of the pancreas appears to be associated with adequate clinical and oncological outcome.

Abbreviations: DGE, delayed gastric emptying; ISGPS, International Study Group of Pancreatic Surgery; NODM, new onset diabetes mellitus; SPR, standard pancreatic resection; POPF, postoperative pancreatic fistula.

Financial support and sponsorship: Nil.

Conflict of interest: Nil.

\section{REFERENCES}

1. Luchini C, Fassan M, Doglioni C et al. (2020): Inflammatory and tumor-like lesions of the pancreas. Pathologica, 112: 197-209.

2. Centeno B, Thomas S (2020): Non-Neoplastic Masses of the Pancreas. Pancreat. Tumors, 26: 42-52.

3. World Health Organization (2020): The International Agency for Research on Cancer (IARC), Globocan 2020: Global Cancer Observatory. https://gco.iarc.fr/today/about

4. Etekpo A, Alghawalby A, Alghawalby M et al. (2018): Differences in MUC4 Expression in Pancreatic Cancers and Pancreatic Cysts in Egypt. J Carcinog. Mutagen, 9: 312-16.

5. Schima W, Böhm G, Rösc C et al. (2020): Mass-forming pancreatitis versus pancreatic ductal adenocarcinoma: CT and MR imaging for differentiation. Cancer Imaging, 20: $52-55$.

6. Kersting S, Janot M, Munding J et al. (2012): Rare Solid Tumors of the Pancreas as Differential Diagnosis of Pancreatic Adenocarcinoma. JOP J Pancreas, 13: 268-277.

7. Basturk O, Askan G (2016): Benign Tumors and Tumorlike Lesions of the Pancreas. Surg Pathol Clin., 9: 619-641.

8. Stauffer J, Asbun H (2018): Rare Tumors and Lesions of the Pancreas. Surg Clin., 98: 169-188.

9. Chen Z, Lin X, Li M et al. (2021): Inflammatory myofibroblastic tumor of the pancreatic neck: A case report and review of literature. World J Clin Cases, 9: 6418-6427.

10. Karoumpalis I, Christodoulou D (2016): Cystic lesions of the pancreas. Ann Gastroenterol Q Publ Hell Soc Gastroenterol., 29: 155-161.

11. Bartolini I, Bencini L, Risaliti M et al. (2018): Current Management of Pancreatic Neuroendocrine Tumors: From Demolitive Surgery to Observation. Gastroenterol Res Pract., 18: 9647247.
12. Eapen A, Chandramohan A, John R et al. (2012): Imaging of an Indeterminate Pancreatic Mass. J Gastrointest Abdom Radiol., 3: 75-86.

13. Philips S, Shah S, Vikram R et al. (2012): Pancreatic endocrine neoplasms: a current update on genetics and imaging. Br J Radiol., 85: 682-696.

14. Ramesh J, Council L, Wilcox C (2013): Recurrent pancreatitis caused by pancreatic ductal villous adenoma treated with endoscopic snare polypectomy. Endoscopy, 45: 23-24.

15. Askew J, Connor S (2013): Review of the investigation and surgical management of resectable ampullary adenocarcinoma. HPB., 15: 829-838.

16. Chua T, Yang T, Gill A et al. (2016): Systematic Review and Meta-Analysis of Enucleation Versus Standardized Resection for Small Pancreatic Lesions. Ann Surg Oncol., 23: 592-599.

17. Beger H, Mayer B, Vasilescu C et al. (2022): Long-term Metabolic Morbidity and Steatohepatosis Following Standard Pancreatic Resections and Parenchyma-sparing, Local Extirpations for Benign Tumor: A Systematic Review and Meta-analysis. Ann Surg., 275: 54-66.

18. Beger H, Mayer B, Poch B (2018): Parenchyma-sparing, local pancreatic head resection for premalignant and lowmalignant neoplasms - A systematic review and metaanalysis. Am J Surg., 216: 1182-1191.

19. Bassi C, Marchegiani G, Dervenis C et al. (2017): The 2016 update of the International Study Group (ISGPS) definition and grading of postoperative pancreatic fistula: 11 Years After. Surgery, 161: 584-591.

20. Cakir M, Akinci M, Akturk O (2020): Delayed Gastric Emptying as a Complication of Whipple's Procedure: Could it be Much Less Frequent than Anticipated? Could the Definition Be Revised? A Single Center Experience. Medeni Med J., 35: 181-187.

21. Lalwani N, Mannelli L, Moorthy D et al. (2015): Uncommon pancreatic tumors and pseudotumors. Abdom Imaging, 40: 167-180.

22. Yadav R, Jiang X, Chen J (2020): Differentiating benign from malignant pancreatic cysts on computed tomography. Eur J Radiol Open, 7: 278-82.

23. Osman H, Jeyarajah D (2020): Pancreas Cystic Lesions. Surg Clin North Am., 100: 581-588.

24. Zhou Y, Zhao M, Wu L et al. (2016): Short- and longterm outcomes after enucleation of pancreatic tumors: An evidence-based assessment. Pancreatol Off J Int Assoc Pancreatol. , 16: 1092-1098.

25. Jilesen A, van Eijck C, Buschet O et al. (2016): Postoperative outcomes of enucleation and standard resections in patients with a pancreatic neuroendocrine tumor. World J Surg., 40: 715-728.

26. Wang X, Chen Y, Tan C et al. (2018): Enucleation of pancreatic solid pseudopapillary neoplasm: Short-term and long-term outcomes from a 7-year large single-center experience. Eur J Surg Oncol., 44: 644-650.

27. Hüttner F, Koessler-Ebs J, Hackert T et al. (2015): Metaanalysis of surgical outcome after enucleation versus standard resection for pancreatic neoplasms. BJS Br J Surg., 102: 1026-1036.

28. Song K, Kim S, Hwang D et al. (2015): Enucleation for benign or low-grade malignant lesions of the pancreas: Single-center experience with 65 consecutive patients. Surgery, 158: 1203-1210.

29. Lubezky N, Papoulas M, Lessing Y et al. (2017): Solid pseudopapillary neoplasm of the pancreas: Management and long-term outcome. Eur J Surg Oncol., 43: 1056-1060. 